\title{
Research on roles played by stromal cell-derived factor 1 and chemokine receptor 4 in diagnosis on proliferation, infiltration and metastasis of cervical cancer.
}

\author{
Feng Feng Cheng ${ }^{1 \#, ~ Y a n ~ K o n g ~}{ }^{1 \# *}$, Aiju Fang ${ }^{1}$, Jing Yu ${ }^{1}$, Xiao Ying Zhang ${ }^{1}$, Yingjie Li ${ }^{2}$ \\ ${ }^{1}$ Department of Obstetrics and Gynecology, Shandong Traffic Hospital, Jinan, PR China \\ ${ }^{2}$ Department of Pathology, Shandong Traffic Hospital, Jinan, PR China \\ \#These authors contributed equally to this work
}

\begin{abstract}
Purpose: To understand the influence of Stromal Cell-Derived Factor 1 (SDF-1) and corresponding receptor CXCR4 on proliferation, infiltration and metastasis of cervical cancer HeLa cells.

Method: The flow cytometry dyeing and the Western blot method are adopted to detect the expression conditions of CXCR4 in HeLa cells; the MTT method is adopted to detect the influence of SDF-1 and corresponding receptor CXCR4 on proliferation of HeLa cells; the Boyden chamber method is adopted to detect the influence of SDF-1 and corresponding receptor CXCR4 on infiltration and metastasis of HeLa cells.

Results: There is high expression on receptor CXCR4, the receptor of SDF-1; after the SDF-1 stimulation, the proliferation ability of HeLa cells is significantly increased $(P<0.05)$, and this effect is dose-dependent; after the knock-down of CXCR4 expression on surface of HeLa cells with RNAi, the proliferation of HeLa cells caused by SDF-1 stimulation is significantly interdicted $(\mathbf{P}<0.05)$. SDF-1 may give rise to infiltration and metastasis of HeLa cells. After the knock-down of CXCR4 expression on surface of HeLa cells with RNAi, the infiltration and metastasis of HeLa cells are significantly interdicted $(\mathbf{P}<\mathbf{0 . 0 5})$.

Conclusion: The CXCR4 with high expression on surface of HeLa cells plays a significant role in inducing proliferation, infiltration and metastasis of HeLa cells.
\end{abstract}

Keywords: Stromal cell-derived factor 1, CXCR4, Cervical cancer, HeLa cells.

Accepted on November 10, 2017

\section{Introduction}

As the commonest gynecologic malignant tumor, cervical cancer is the second largest reason for death of women in developing countries [1,2]. The prognosis of cervical cancer patients is closely related to clinical stages and pathological categories, and the cervical cancer patients with lymphatic metastasis have poorer prognosis than that without lymphatic metastasis [3]. According to research findings, Stromal CellDerived Factor 1 (SDF-1) and corresponding receptor CXCR4 are closely related to metastasis of multiple tumors [4,5], and there is little research on roles of SDF-1 and corresponding receptor CXCR4 in cervical cancer. Therefore, HeLa cells of human cervical carcinoma are adopted as the research model in this experiment, to conduct preliminary discussion on roles played by SDF-1 and corresponding receptor CXCR4 in inducing of proliferation, infiltration and metastasis of Hela cells. According to research findings, there is high CXCR4 expression on surface of Hela cells, and SDF-1 simulation can give rise to proliferation, infiltration and metastasis of HeLa cells. After the knock-down of the expression of CXCR4 on HeLa cell surface, the proliferation, infiltration and metastasis caused by SDF-1 stimulation are significantly interdicted. It indicates that the CXCR4 with high expression on surface of HeLa cells plays a significant role in inducing proliferation, infiltration and metastasis of HeLa cells.

\section{Data and Method}

\section{Material}

The human cervical carcinoma HeLa cells were purchased from ATCC; the chromosome antibodies of CXCR4 flow cytometry were purchased from Biolegend Company; the CXCR4 Western blot antibodies were purchased from R\&D Company; the CXCR4 siRNAs were purchased from Santa Cruz Company; the electric transfection kit was purchased from Lonza Company; the Boyden chamber was purchase from American Millipore Company; the CytoBuster protein extraction reagent was purchased from Novagen Company; the 
Research on roles played by stromal cell-derived factor 1 and chemokine receptor 4 in diagnosis on proliferation, infiltration and metastasis of cervical cancer

protease and phosphatase inhibitor were purchased from Thermo Company.

\section{Cell culture}

The cervical cancer cell strains HeLa was cultivated with RPMI1640 culture medium with $10 \%$ fetal calf serum in conditions of $37^{\circ} \mathrm{C}$ and $5 \% \mathrm{CO}_{2}$. The passage was conducted at the time of $80 \%$ integration of cells.

\section{The flow cytometry dyeing method}

Hela cells were washed twice by PBS with $1 \%$ BSA, and corresponding fluorescent antibodies (CXCR4) were added, for incubation of $30 \mathrm{~min}$ at $4{ }^{\circ} \mathrm{C}$ keeping out of the sun; they were washed twice by PBS with $1 \%$ BSA, and then the cells were re-suspended in $0.1 \mathrm{ml} \mathrm{1 \%}$ paraformaldehyde stationary liquid for detection of flow cytometry.

\section{The extraction of total cell protein}

Hela cells were washed twice by iced PBS, $400 \mathrm{Xg}$ centrifuged for $5 \mathrm{~min}$; the decellularization supernatant was abandoned, and then protease, phosphatase inhibitor and $100 \mu \mathrm{L}$ CytoBuster protein extraction reagent were added into the sediment, which were blown and beat for mixing, and was placed for $15 \mathrm{~min}$ at room temperature; $4^{\circ} \mathrm{C}, 12000 \mathrm{Xg}$ centrifuged for $15 \mathrm{~min}$; the acquired supernatant was the total cell protein, some of which was kept for detection on protein concentration, and the other supernatant was sub-packaged with $15 \mu \mathrm{L}$ in each pipe, for freeze preservation at $-80^{\circ} \mathrm{C}$.

\section{Measurement of protein concentration with the BCA method}

The dilution was conducted on the standard substance BSA strictly according to the specification of the BCA kit; Reagent $\mathrm{A}$ and $\mathrm{B}$ in the kit were mixed according to the volume ratio of $50: 1$, to acquire the working solution; $200 \mu \mathrm{L}$ working solution was added into the 96-well plate, and then $25 \mu \mathrm{L}$ multiple proportion diluted standard substance or sample to be tested was added, and 3 multiple pores were set for each category of sample. The OD value was measured at the wave length of 562 $\mathrm{nm}$ after incubation at $37^{\circ} \mathrm{C}$ for $30 \mathrm{~min}$. The standard curve was drawn according to the optical density and the concentration gradient of the standard substance, and the concentration of the sample was calculated according to the standard curve and the OD value of the sample to be tested.

\section{Western blot}

The above protein of the same amount was extracted, which was transfected and printed half-dry after $8 \%-10 \%$ SDS-PAGE separation gel and 5\% spacer gel separation. The TBST with $5 \%$ BSA was sealed and incubated for $2 \mathrm{~h}$ at indoor temperature, and then mouse anti-human CXCR4 antibodies were added for incubation overnight at $4^{\circ} \mathrm{C}$. On the next day, it was conducted with membrane cleaning for 3 times with $0.1 \%$ TBST, 5 min each time. The HRP of corresponding species was added to mark the second antibody, for incubation of $1 \mathrm{~h}$ at indoor temperature. It was then conducted with membrane cleaning with $0.1 \%$ TBST, and Supersignal West Femto/Pico HRP sensitive chemiluminiscence substrate was adopted for color development to the stripe. Actin was adopted as the internal control. All the experiments should be conducted at least 3 times.

\section{Procedures and effect detection of transfection}

Please refer to the specification of the kit for detailed operating procedures.

\section{Detection on cell proliferation ability with the MTT method}

The RPMI-1640 culture medium with low serum was adopted to cultivate for Hela cells, and SDF-1 of $0,1,10,20,50,100$ and $200 \mathrm{ng} / \mathrm{ml}$ was added into the culture medium. The cells were inoculated into 96-core culture plate. After $72 \mathrm{~h}$ of cultivation, each hole was added into $20 \mu \mathrm{L}$ MTT solution, and the supernatant was extracted after action of $4 \mathrm{~h}$. The DMSO solution of $150 \mu \mathrm{L}$ was added for $10 \mathrm{~min}$ of low velocity vibration, and the OD value was measured at the wave length of $490 \mathrm{~nm}$.

\section{Detection on infiltration ability}

The Boyden chamber was adopted to analyse and detect cell infiltration ability, with the following procedures: The trypan blue was adopted for counting and re-suspending of cells for experiments. $1 \times 10^{5}$ cells were placed in upper layer chamber spread with glue, and the culture medium was supplemented to $300 \mu \mathrm{L}$. The cotton swab was adopted to eliminate the cells without infiltration from the upper layer chamber. The cells in lower layer chamber were fixed and dyed with $0.1 \%$ crystal violet, after incubation of $12 \mathrm{~h}$, so as to count the number of cells with infiltration.

\section{Statistical method}

The SPSS 16.0 software was adopted, and t-test was adopted for statistical analysis on the data. The difference had statistical significance if $\mathrm{P}<0.05$.

\section{Results}

\section{Detection on CXCR4 expression on surface of HeLa cells of cervical cancer}

As shown in Figure 1, according to the detection result of the flow cytometry, CXCR4 receptor expression rate on surface of HeLa cells of cervical cancer was as high as more than $90 \%$. At the same time, according to Western blot results, the CXCR4 receptor had high expression on HeLa cells in cervical cancer. 


\section{Detection on cell transfection efficiency and interfering effect}

As shown in Figure 2A, the Lonza electric transfection method was adopted for HeLa cell strain transfection of cervical cancer, with transfection ratio of more than 90\%; after the transfection of CXCR4 siRNA, the positive rate of CXCR4 expression on surface of HeLa cells of cervical cancer was significantly reduced (Figure 2B).
A

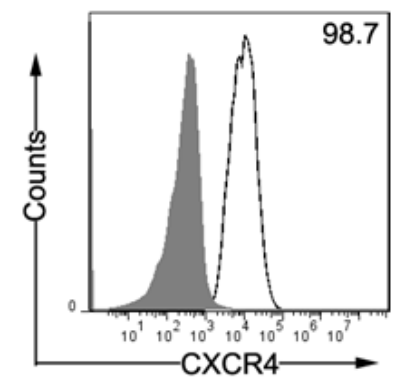

B

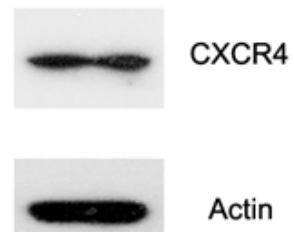

Actin
Figure 1. Results on analysis in CXCR4 expression in HeLa cells.
A

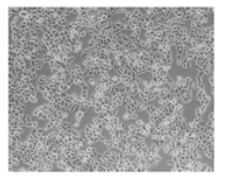

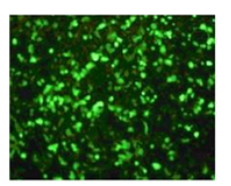

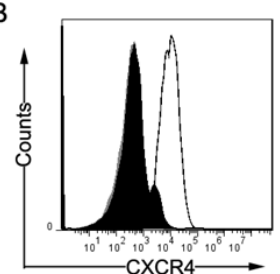

Figure 2. Detection on cell transfection efficiency and interfering effect.

\section{Influence of SDF-1 and corresponding receptor CXCR4 on HeLa cell proliferation}

As shown in Figure 3A, after the treatment of Hela cells with SDF-1 of $0,1,10,20,50,100$ and $200 \mathrm{ng} / \mathrm{ml}$, the proliferation efficiency of Hela cells was $(0.358 \pm 0.124),(0.685 \pm 0.176)$, $(1.056 \pm 0.135),(1.524 \pm 0.204),(1.867 \pm 0.267),(2.157 \pm$ $0.301)$ and $(2.025 \pm 0.287)$, respectively. With the increase in SDF-1 concentration, the proliferation efficiency of Hela cells gradually increased; at the SDF-1 concentration of $100 \mathrm{ng} / \mathrm{ml}$, the proliferation efficiency of Hela cells achieved the highest. After the knock-down of the CXCR4 expression on surface of HeLa cells with RNAi, the HeLa cell proliferation caused by SDF-1 stimulation was significantly interdicted $(\mathrm{P}<0.05)$ (Figure 3B).

\section{Influence of Transwell detection SDF-1 and corresponding receptor $\mathrm{CXCR} 4$ on infiltration and metastasis of HeLa cells}

As shown in Figure 4A, the number of cells with infiltration on Hela without any treatment was $(100.25 \pm 19.46)$. After the SDF-1 stimulation, the number of invaded cells in Hela cells was $(304.25 \pm 33.24)$, and the number of the invaded cells in
Hela cells after knockdown of CXCR4 expression group on surface of HeLa cells was (103.24 \pm 22.34$)$. The number of invaded cells after SDF-1 stimulation after knockdown of CXCR4 expression on surface of HeLa cells with RNAi was $(114.45 \pm 24.24)$. It indicates that after the knockdown of CXCR4 on surface of HeLa cells, the HeLa cell infiltration caused by SDF-1 was significantly interdicted $(\mathrm{P}<0.05)$.
A

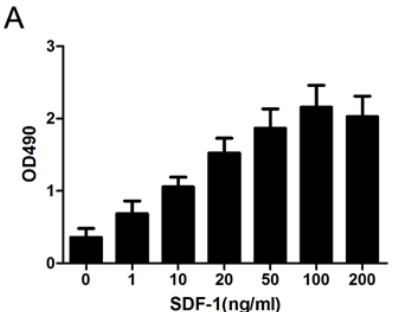

B

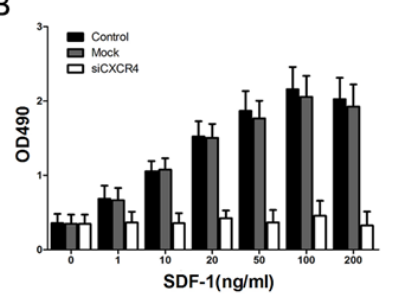

Figure 3. Detection results on proliferation rate of HeLa cells of cervical cancer.

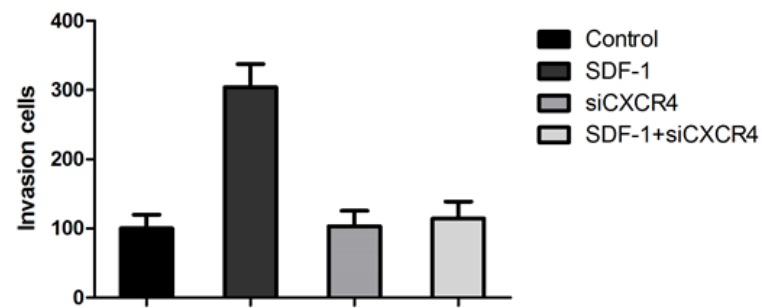

Figure 4. Detection on HeLa cell infiltration and metastasis ability.

\section{Discussion}

As the commonest gynecological malignant tumor, cervical cancer is the second largest reason for death of women in developing countries [1,2]. The prognosis of cervical cancer patients is closely related to clinical stages and pathological categories, and the cervical cancer patients with lymphatic metastasis have poorer prognosis than that without lymphatic metastasis [3]. Chemotactic factors play a main role of promoting cell migration. Cells migrate to the places with high concentration of chemotactic factors along with the signal on increased chemotactic factor concentration. According to research findings, multiple chemotactic factors and related receptors participate in infiltration and metastasis of tumor cells, playing significant roles in occurrence and development of tumors. CXCR4 is an important member in chemokine receptor family. According to research findings, CXCR4 is closely related to growth, infiltration and metastasis of breast cancer, gastric cancer, colorectal cancer, pancreatic duct cell cancer and liver cancer [6-10]. Based on research findings, HeLa cells of cervical cancer have relatively high level on CXCR4 expression. At the same time, the corresponding chemotactic factors in CXCR4 in cervical cancer tissues, Stromal Cell-Derived Factor 1 (SDF-1), have relatively high expression [11]. It indicates that CXCR4 plays important roles in occurrence and development of cervical cancer. In order to discuss on the mechanism on occurrence and development of cervical cancer, the influence of $\mathrm{CXCR} 4$ and SDF-1 on 
proliferation, infiltration and metastasis on HeLa cells of cervical cancer have been observed. Based on results, with SDF-1 stimulation of different concentrations in Hela cell culture medium can significantly improve the proliferation ability of SDF-1 $(\mathrm{P}<0.05)$. At the same time, SDF-1 can also give rise to infiltration and metastasis of HeLa cells. After the knock-down of CXCR4 expression on surface of HeLa cells with RNAi, the infiltration and metastasis of HeLa cells caused by SDF-1 stimulation are significantly interdicted $(\mathrm{P}<0.05)$. Therefore, CXCR4 with high expression on surface of HeLa cells plays important role in inducing proliferation, infiltration and metastasis of HeLa cells, and CXCR4 may participate in occurrence and development of cervical cancer by promoting proliferation, infiltration and metastasis of HeLa cells. However, the mechanism for CXCR4 and corresponding chemotactic factors to induce the proliferation, infiltration and metastasis of HeLa cells shall be further researched.

\section{Acknowledgement}

This work was funded by Natural Science Foundation of China Youth Fund (ZR2013HQ062).

\section{References}

1. Chen A, Wang Y, Zhang X. Recall rate of positive people with cervical cancer screening and analysis on the influencing factors. Chinese J Publ Health 2014; 1098181.

2. Zhang Y, Wei J. Research progress on cervical cancer screening and progress tumor markers. Chinese J Publ Health 2013; 28: 374-376.

3. Sun $\mathrm{W}$, Cai S, Yan X. Influence of CXCR7 on proliferation, adherence and infiltration ability of HeLa cells. J Univ Sci Technol China 2012; 42: 311-317.

4. Rhee YH, Chung PS, Kim SH. CXCR4 and PTEN are involved in the anti-metastatic regulation of anethole in DU145 prostate cancer cells. Biochem Biophys Res Commun 2014; 447: 557-562.

5. Liu H, Zhou W, Pan Z. Functions of chemokine receptor CXCR4 and ligand CXCL12 in hepatoma carcinoma cell migration. Chinese J Exp Surg 2008; 25: 1363-1365.
6. Chen HW, Du CW, Wei XL, Khoo US. Cytoplasmic CXCR4 high-expression exhibits distinct poor clinicopathological characteristics and predicts poor prognosis in triple-negative breast cancer. Curr Mol Med 2013; 13: 410-416.

7. Satomura H, Sasaki K, Nakajima M. Can expression of CXCL12 and CXCR4 be used to predict survival of gastric cancer patients? Anticancer Res 2014; 34: 4051-4057.

8. Heckmann D, Maier P, Laufs S. The disparate twins: a comparative study of CXCR4 and CXCR7 in SDF-1 $\alpha$ induced gene expression, infiltration and chemosensitivity of colon cancer. Clin Cancer Res 2014; 20: 604-616.

9. Arora S, Bhardwaj A, Singh S. An undesired effect of chemotherapy: gemcitabine promotes pancreatic cancer cell invasiveness through reactive oxygen speciesdependent, nuclear factor $\kappa \mathrm{B}$ - and hypoxia-inducible factor $1 \alpha$-mediated up-regulation of CXCR4. J Biol Chem 2013; 288: 21197-211207.

10. Ghanem I, Riveiro ME, Paradis V. Insights on the CXCL12-CXCR4 axis in hepatocellular carcinoma carcinogenesis. Am J Transl Res 2014; 6: 340-352.

11. Jin F, Brockmeier U, Otterbach F. New insight into the SDF-1/CXCR4 axis in a breast carcinoma model: hypoxia-induced endothelial SDF-1 and tumor cell CXCR4 are required for tumor cell intravasation. Mol Cancer Res 2012; 10: 1021-1031.

\section{*Correspondence to}

Yan Kong

Department of Obstetrics and Gynecology

Shandong Traffic Hospital

PR China 